\title{
Periodic Behavior of the Exciton Oscillator Strength with AlAs Thickness in Type II GaAs/AlAs Heterostructures
}

\author{
C. Gourdon, D. Martins, P. Lavallard \\ Groupe de Physique des Solides, Universités Paris 6 et Paris 7 \\ CNRS UMR 75-88, Tour 23, 2 Place Jussieu, 75251 Paris cedex 05, France
}

AND E.L. IVCHENKO

Ioffe Physico-technical Institute, Russian Academy of Sciences

194021 St Petersburg, Russia

\begin{abstract}
For a single GaAs/AlAs/GaAs type II pseudodirect double quantum well, as well as for superlattices it was predicted that the oscillator strength of the lowest optical transition has a periodic dependence on the number of AlAs monolayers. The oscillator strength depends on the coupling between the $\Gamma$ and $X$ electron states. We use samples containing a single GaAs/AlAs/GaAs double quantum well with thickness gradient to show experimental evidence of this effect. The results concerning the $\Gamma-X$ coupling are obtained from the study of the ratio of photoluminescence intensities of the zero-phonon line and the phonon replica and from their time decay. They show the monolayer dependence of the $\Gamma-X$ mixing potential. We extend the model describing the $\Gamma-X$ coupling for ideal interfaces in the frame of the envelope approximation to the case of non-abrupt interfaces and exciton localization. The amplitude of variation of the radiative recombination time due to the $\Gamma-X$ mixing is well reproduced within this model.
\end{abstract}

PACS numbers: 78.66.-w, 71.70.-d

\section{Introduction}

Type II GaAs/AlAs heterostructures have been the subject of a number of experimental [1-7], as well as theoretical investigations [8-11] in the past years. 
In such structures the electron and the hole are confined in adjacent layers. The oscillator strength of the lowest optical transition depends on the overlap of the electron and the hole envelope functions. The spatially indirect character of this optical transition makes it possible to use the exciton as a probe of interface-related properties of the excitonic states [6]. In GaAs/AlAs type II heterostructures, the lowest optical transition is not only indirect in real space but also in $k$-space. The electron wave function is derived from a zone-edge $X$-state of AlAs. One of the most surprising consequences is that for structures which are symmetrical with respect to the middle of an AlAs slab (superlattices for instance), the oscillator strength of the lowest optical transition should depend on the parity of the number $M$ of AlAs monolayers (ML) in the AlAs slabs [8-11]. Due to the interplay between confinement and strain effects, the lowest electronic state can be either of $X_{z}$-type or of $X_{x y}$-type ( $z$ denotes the growth axis). Structures with $X_{z}$ as lowest state are named pseudodirect since the $X_{z}$ state can be coupled to the electron $\Gamma$ state at interfaces. For even $M$, the contributions to the coupling of successive GaAs/AlAs and AlAs/GaAs interfaces add up and the optical transition is allowed whereas, for odd $M$, these contributions cancel out and the transition is forbidden. This effect arises from the symmetry properties of the zone-edge $X_{z}$-Bloch function under symmetry operations that change $z$ into $-z$.

Layer thickness fluctuations is a major problem to investigate monolayer-dependent properties. Previous attempts to show experimental evidence of the parity-dependent optical selection rule using superlattices either were unsuccessful [5] or led to contradictory results $[12,13]$. Our choice was to use a single $\mathrm{GaAs} / \mathrm{AlAs} / \mathrm{GaAs}$ type II double quantum well (DQW). Absorption is quite weak, therefore only photoluminescence (PL) can be used to show evidence of the periodic behavior of the exciton oscillator strength with AlAs thickness. We report hereafter on PL and time-resolved PL results.

\section{Samples}

The samples were grown by molecular beam epitaxy at Laboratoire de Microstructures et Microélectronique (Bagneux, France). A large gradient of AlAs thickness across the wafer is achieved by stopping the rotation of the substrate during the growth of $\mathrm{AlAs}$ in the DQW. Typical GaAs and AlAs thicknesses are between 3 and 7 MLs ( 1 monolayer (ML) corresponds to $2.83 \AA$ ). With thicker layers the parity effect can no longer be observed. Additional quantum wells are grown in the structure to provide accurate calibration of GaAs and AlAs thickness by optical spectroscopy and electronic microscopy. Details on the growth conditions and thickness calibration can be found elsewhere [14]. Samples are cleaved from 2-inch wafers in the form of rectangular pieces of length $50 \mathrm{~mm}$ and width 3-4 mm. Sample 17Q55 consists of a DQW with nominal structure at the center of the wafer $\operatorname{GaAs}(6 \mathrm{ML}) / \operatorname{AlAs}(6 \mathrm{ML}) / \operatorname{GaAs}(6 \mathrm{ML})$ embedded in $1000 \AA$ of 
$\mathrm{Ga}_{0.6} \mathrm{Al}_{0.4} \mathrm{As}$ alloy layers. Unfortunately, the DQW was found to be asymmetrical, the first GaAs layer being thinner than the second one by $1.4 \mathrm{ML}$. This asymmetry originates from Ga evaporation during the growth interruption and temperature rise at the GaAs/AlAs interface. For sample $39 \mathrm{Z} 36$ the composition is similar but the substrate temperature was kept constant for GaAs and AlAs growth in order to prevent Ga evaporation.

PL was excited with the $514 \mathrm{~nm}$ line of an argon ion laser. For time-resolved $\mathrm{PL}$, laser pulses were generated with a $250 \mathrm{MHz}$ acousto-optical modulator. $50 \mathrm{~ns}$ pulses with a $50 \mu$ s period were used. PL was detected with a time-correlated single photon counting system.

\section{Results and model}

\subsection{Photoluminescence}

PL spectra consist of a zero-phonon line ( $\mathrm{ZPH})$ and phonon replica $(\mathrm{PH})$. In order to determine the dependence of the $\Gamma-X$ coupling matrix element on AlAs thickness we have studied the ratio $I_{\mathrm{zph}} / I_{\mathrm{ph}}$ of the intensities of the $\mathrm{ZPH}$ line and the LO PH replica. Contrary to the $\Gamma-X$ coupling the electron-phonon interaction is independent of the parity of the number $M$ of AlAs MLs since the symmetry of the LO phonon state changes with $M$ in the same manner as the symmetry of the electron Bloch $X$-state [15]. In the first approach the ratio $I_{\mathrm{zph}} / I_{\mathrm{ph}}$ is equal to $w_{\Gamma X} / w_{\mathrm{ph}}$, where $w_{\Gamma X}$ is the radiative recombination rate due to the $\Gamma-X$ coupling and $w_{\mathrm{ph}}$ is the recombination rate due to the LO-phonon assisted transition. In second-order perturbation theory, one has

$$
\left(\frac{I_{\mathrm{zph}}}{I_{\mathrm{ph}}}\right)_{\mathrm{calc}}=\left|\frac{M_{\Gamma X}}{M_{\mathrm{el}-\mathrm{ph}}} \frac{E_{\Gamma}-E_{X}+h \nu_{\mathrm{ph}}}{E_{\Gamma}-E_{X}}\right|^{2},
$$

where $E_{\Gamma}, E_{X}$ are the energies of the $H H_{1}-\Gamma_{1}$ and $H H_{1}-X_{1}$ excitonic transitions, respectively, and $h \nu_{\mathrm{ph}}$ is the LO phonon energy. $M_{\Gamma X}$ and $M_{\mathrm{el}-\mathrm{ph}}$ are the matrix elements of the $\Gamma-X$ coupling and the electron-phonon interaction, respectively. In order to show that the $\Gamma-X$ coupling depends on $M$, one must compare the experimental ratio $I_{\mathrm{zph}} / I_{\mathrm{ph}}$ with the ratio calculated with a constant value of $\left|M_{\Gamma X} / M_{\mathrm{el}-\mathrm{ph}}\right|^{2}$. The $E_{\Gamma}$ energy is obtained from the weak $H H_{1}-\Gamma_{1} \mathrm{PL}$ line observed when the excitation intensity is increased. The ratios $I_{\mathrm{zph}} / I_{\mathrm{ph}}$ and $\left(I_{\mathrm{zph}} / I_{\mathrm{ph}}\right)_{\text {calc }}$ are displayed in Fig. 1 for sample 17Q55. Their different behaviors with AlAs thickness give a clear indication of the monolayer dependence of the $\Gamma-X$ coupling. Due to the asymmetry of the DQW the ratio $I_{\mathrm{zph}} / I_{\mathrm{ph}}$ varies only by a factor 1.7 between odd and even $M$. The amplitude of variation is larger (2.4) for the more symmetrical DQW of sample 39736 (Fig. 2). The periodic behavior of $I_{\mathrm{zph}} / I_{\mathrm{ph}}$ is also more clearly seen owing to the larger AlAs gradient. The ratio $I_{\mathrm{zph}} / I_{\mathrm{ph}}$ shows minima at positions where the average AlAs thickness is about 6 and 4 MLs. It seems that the observed dependence of the oscillator strength on 


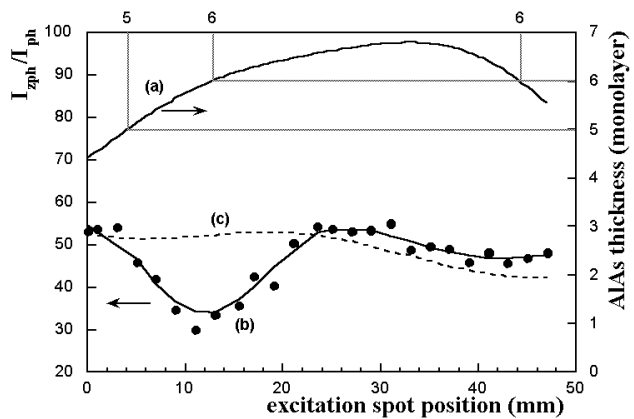

Fig. 1. $a-\mathrm{AlAs}$ thickness for the DQW of sample 17Q55 as a function of the position of the excited spot along the sample, $b$ - experimental ratio $I_{\mathrm{zh}} / I_{\mathrm{ph}}$ (black circles, the solid line is a guide for the eyes) and $c$ - calculated ratio $\left(I_{\mathrm{zph}} / I_{\mathrm{ph}}\right)_{\mathrm{calc}}$ (dashed line) with a constant ratio $\left|M_{\Gamma X} / M_{\mathrm{el}-\mathrm{ph}}\right|^{2}$ taken as an adjustable parameter.

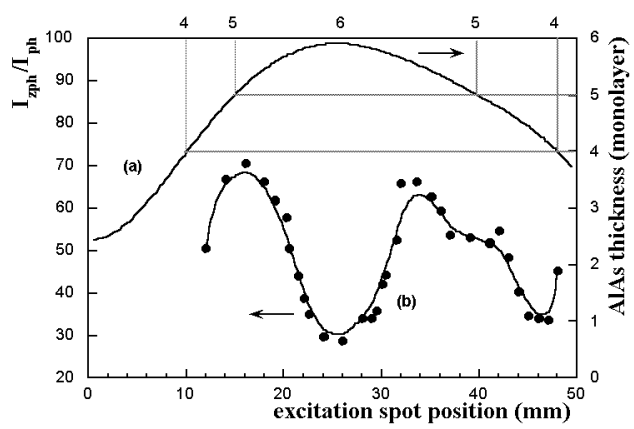

Fig. 2. $\quad a-$ AlAs thickness for the DQW of sample $39 \mathrm{Z} 36$ as a function of the position of the excited spot along the sample, $b$ - experimental ratio $I_{\mathrm{zph}} / I_{\mathrm{ph}}$ (black circles). The solid line is a guide for the eyes. This ratio cannot be obtained between positions 0 and $10 \mathrm{~mm}$. In this region the disappearance of $\mathrm{PH}$ replica and the variation of the ZPH line with sample composition indicate a type II to type I transition.

the parity of $M$ is opposite to the theoretical one. This is attributed to exciton localization. The ZPH PL line is $6-10 \mathrm{meV}$ broad. Its energy varies smoothly with sample composition. Excitons are very likely localized in regions where the effective AlAs thickness is larger than the average thickness determined by the calibration procedure which is relative to the free exciton. An effective AlAs thickness for localized excitons larger than the average one by $\approx 1 \mathrm{ML}$ is consistent with the line width (which is usually of the same order as the Stokes shift resulting from localization). Hence our results are in accordance with the theoretical predictions.

\subsection{Time-resolved photoluminescence}

Time-resolved PL measurements were performed to obtain separately the radiative recombination rates $w_{\Gamma X}$ and $w_{\mathrm{ph}}$. The $\mathrm{PL}$ decay is found to be non- 
-exponential both in the ZPH line and in the $\mathrm{PH}$ replica. This is attributed to a distribution $p\left(w_{\Gamma X}\right)$ of the $w_{\Gamma X}$ recombination rate for localized excitons. From the analysis of the decay of the PL signal both in the ZPH line and in the replica we obtain the radiative decay time due to the $\Gamma-X$ coupling $\tau_{\Gamma X}=\left(w_{\Gamma X}\right)^{-1}$ and the decay time due to phonon-assisted transition $\tau_{\mathrm{ph}}=\left(w_{\mathrm{ph}}\right)^{-1}$. We have neglected the nonradiative recombination [16]. $\tau_{\Gamma X}$ and $\tau_{\mathrm{ph}}$ are plotted in Fig. 3 for sample $17 \mathrm{Q} 55 . \tau_{\mathrm{ph}}$ is in the range $80-150 \mu \mathrm{s}$. The solid line represents the recombination time calculated in second-order perturbation theory [14]. The increase in $\tau_{\mathrm{ph}}$ across the sample is mainly due to the increase in $E_{\Gamma}-E_{X}$.

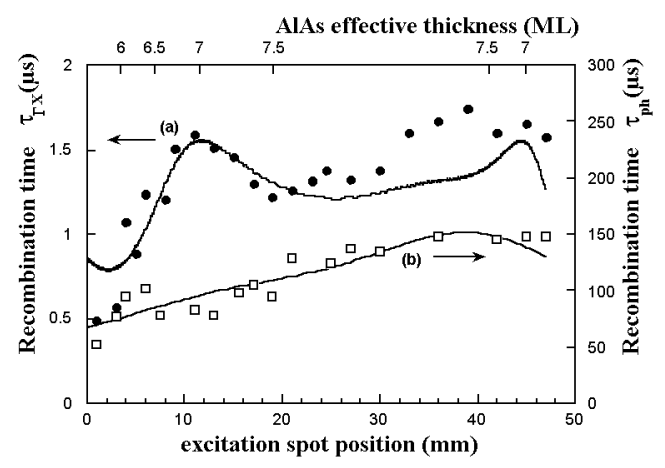

Fig. 3. Sample 17Q55: $a$ - radiative time $\tau_{\Gamma X}$ due to the $\Gamma-X$ coupling (experimental: circles, calculated: solid line) as a function of position of the excited spot along the sample; $b$ - recombination time for the phonon-assisted transition $\tau_{\mathrm{ph}}$ (experimental: squares, calculated: solid line). The effective AlAs thickness for localized excitons (see text) is shown at the top of the figure.

$\tau_{\Gamma X}$ varies between 0.5 and $1.7 \mu \mathrm{s}$. In agreement with the time-integrated data we observe a maximum of $\tau_{\Gamma X}$ around the position $10 \mathrm{~mm}$ and possibly around $40 \mathrm{~mm}$, where the ratio $I_{\mathrm{zph}} / I_{\mathrm{ph}}$ shows minima. The behaviors of $\tau_{\Gamma X}$ and $\tau_{\mathrm{ph}}$ are clearly different. This confirms the monolayer dependence of the $\Gamma-X$ coupling. In the next section we develop a model describing the $\Gamma-X$ coupling for localized excitons. Within this model we are able to reproduce the amplitude of variation of $\tau_{\Gamma X}$.

\subsection{Model}

The generalized envelope-function approximation has been applied to describe the $\Gamma-X$ coupling in the case of ideally plane interfaces [11]. The $\Gamma-X$ mixing potential acting on the envelope functions is a sum of $\delta$-functions at each interface

$$
V_{\Gamma X}(z)=\sum_{i} t_{\Gamma X}\left(z_{i}\right) a V \delta\left(z-z_{i}\right)
$$


The interfaces are taken as As planes at positions $z_{i} . V$ is equal to $\hbar^{2} / 2 m_{0} a^{2}$, where $a$ is the lattice constant and $m_{0}$ is the free electron mass. $t_{\Gamma X}\left(z_{i}\right)$ is equal to $t_{\Gamma X} \eta\left(z_{i}\right)$, where $t_{\Gamma X}$ is a dimensionless coupling parameter and $\eta\left(z_{i}\right)$ is the phase factor of the Bloch zone-edge wave function. It can be written as $\eta\left(z_{i}\right)=\cos \left(2 \pi z_{i} / a\right)=(-1)^{2 z_{i} / a}$. We have extended this model to calculate the $\Gamma-X$ coupling in the case of interface roughness and exciton localization. In a real GaAs/AlAs structure the interface can hardly be defined as an ideal As plane. Fluctuations of the interface position over one or two MLs, when averaged over a certain in-plane distance along the layer plane, result in an effective interface position which can be located anywhere between two As planes. A non-abrupt interface is modelled here by considering one $\mathrm{ML}$ of $\mathrm{Ga}_{1-\xi} \mathrm{Al}_{\xi} \mathrm{As}$ alloy between a pure GaAs layer and a pure AlAs layer. We define an equivalent abrupt interface at position $z_{i}=z_{n}+1-\xi$, where $z=z_{n}$ is the position of the As plane after the last pure Ga plane (in units of $1 \mathrm{ML}$, i.e. a/2). We introduce the coupling coefficient $t_{\Gamma X}\left(z_{i}\right)$ as a function of the position of the effective interface. It continuously varies between $+t_{\Gamma X}$ and $-t_{\Gamma X}$, its values for perfect interfaces at an As plane. In the following we shall take $t_{\Gamma X}(z)$ as $t_{\Gamma X} \cos (\pi z)$, which is the first term of its expansion in the Fourier series.

We assume that this model can be applied to a localized exciton, as well as to a free exciton, since small-scale fluctuations of the interface position are averaged over the relative motion of the electron and the hole. We calculate the $\Gamma-X$ coupling for a localized exciton as if the exciton were free with the electron located in an AlAs layer of effective thickness $e$ which can be different from an integer number of ML. Moreover, at each position $y$ along the sample, the PL line is the sum of the contributions of excitons localized in different sites with position $z_{\mathrm{L}}(y)$ of the left interface and $z_{\mathrm{R}}(y)$ of the right interface distributed around their mean values $\bar{z}_{\mathrm{L}}(y)$ and $\bar{z}_{\mathrm{R}}(y)=\bar{z}_{\mathrm{L}}(y)+\bar{e}(y) \cdot \bar{e}(y)$ is taken as the average AlAs thickness determined by the calibration augmented by 1.1 ML to take into account exciton localization. $\bar{z}_{\mathrm{L}}(y)$ is taken as the $y$-dependent position of the GaAs/AlAs interface of the DQW calculated by adding the thicknesses of the calibration structure, the alloy layer and the first GaAs layer of the DQW. Even with a perfectly flat substrate surface, after the growth of the calibration structure and the $\mathrm{Ga}_{0.6} \mathrm{Al}_{0.4} \mathrm{As}$ alloy layer with (non-intentional) thickness gradient, the sample surface is no more flat but convex. There is a difference of height of about $200 \mathrm{ML}$ from the center to the edge of the wafer.

The $\Gamma-X$ coupling matrix element is then written as

$$
\begin{gathered}
M_{\Gamma X}(y)=t_{\Gamma X} a V\left\{\Psi_{\Gamma}^{\mathrm{L}}(y) \Psi_{X}^{\mathrm{L}}(y) \cos \left[\pi z_{\mathrm{L}}(y)\right]\right. \\
\left.+\Psi_{\Gamma}^{\mathrm{R}}(y) \Psi_{X}^{\mathrm{R}}(y) \cos \left[\pi z_{\mathrm{L}}(y)+\pi e(y)\right]\right\}
\end{gathered}
$$

where $\Psi$ denotes the envelope function. The oscillations of $M_{\Gamma X}(y)$ are averaged by taking into account a distribution of the positions of the interfaces under each excited spot at position $y$. The best agreement with the experimental data is 
obtained by considering uncorrelated Gaussian fluctuations of $z_{\mathrm{L}}$ and $e$ [14]. The distributions of $P_{\mathrm{L}}\left(z_{\mathrm{L}}\right)$ and $P_{e}(e)$ are both taken as Gaussian profiles of width $1 \mathrm{ML}$. The average squared matrix element for the $\Gamma-X$ coupling is calculated at each $y$-position as

$$
\begin{aligned}
& \left|U_{\Gamma X}\right|_{\mathrm{av}}^{2}=\left|\frac{M_{\Gamma X}}{t_{\Gamma X} a V}\right|^{2} \\
& \quad=\iint \mathrm{d} z_{\mathrm{L}} \mathrm{d} e\left[\Psi_{\Gamma}^{\mathrm{L}} \Psi_{X}^{\mathrm{L}} \cos \pi z_{\mathrm{L}}+\Psi_{\Gamma}^{\mathrm{R}} \Psi_{X}^{\mathrm{R}} \cos \pi\left(z_{\mathrm{L}}+e\right)\right]^{2} P_{\mathrm{L}}\left(z_{\mathrm{L}}\right) P_{e}(e) .
\end{aligned}
$$

We calculate the radiative decay time using second-order perturbation theory. The calculated $\tau_{\Gamma X}$ is shown in Fig. 3 as a solid line. The behavior and the amplitude of variation of the experimental values of $\tau_{\Gamma X}$ are quite well reproduced. Taking for the radiative time of the $H H_{1}-\Gamma$ direct transition a value of $0.5-1 \mathrm{~ns}$, we find the dimensionless coupling parameter $\tau_{\Gamma X}$ in the range $0.2-0.3$. This is in good agreement with other experimental determinations [7] and of the right order of magnitude compared to the theoretically calculated one [9]. The $\Gamma-X$ coupling potential is found in the range $1.7-2.5 \mathrm{meV}$.

\section{Conclusion}

The aim of this work was to show experimental evidence of the periodic dependence of the oscillator strength of the lowest optical transition on the number of AlAs MLs in type II pseudodirect GaAs/AlAs heterostructures. In GaAs/AlAs/ GaAs DQW structures with thickness gradient we have studied the ratio of PL intensities of the ZPH line and the LO phonon replica, as well as the time decay of these lines. We have obtained the average radiative recombination time $\tau_{\Gamma X}$ due to the $\Gamma-X$ coupling and the recombination time due to phonon-assisted transition $\tau_{\mathrm{ph}}$ as a function of AlAs thickness. The variation of the $\Gamma-X$ coupling with AlAs thickness clearly shows the ML dependence of the $\Gamma-X$ mixing potential and its oscillatory behavior with AlAs thickness.

We have developed a model describing the $\Gamma-X$ coupling for excitons localized in regions with fluctuations of the position of interfaces. The amplitude of variation of the experimental radiative time $\tau_{\Gamma X}$ is well reproduced within this model.

\section{References}

[1] E. Finkman, M.D. Sturge, M.H. Meynadier, R.E. Nahory, M.C. Tamargo, D.M. Hwang, C.C. Chang, J. Lumin. 39, 57 (1987).

[2] K.J. Moore, P. Dawson, C.T. Foxon, Phys. Rev. B 38, 3368 (1988).

[3] D. Scalbert, J. Cernogora, C. Benoit à la Guillaume, M. Maaref, F.F. Charfi, R. Planel, Solid State Commun. 70, 945 (1989). 
[4] H.W. van Kesteren, E.C. Cosman, P. Dawson, K.J. Moore, C.T. Foxon, Phys. Rev. B 39, 13426 (1989).

[5] Weikun Ge, W.D. Schmidt, M.D. Sturge, L.N. Pfeiffer, K.W. West, J. Lumin. 59, 163 (1994).

[6] C. Gourdon, I.V. Mashkov, P. Lavallard, R. Planel, Phys. Rev. B 57, 3955 (1998).

[7] V. Voliotis, R. Grousson, P. Lavallard, E.L. Ivchenko, A.A. Kiselev, Phys. Rev. B 49, 2576 (1994).

[8] L.J. Sham, Yan-Ten Lu, J. Lumin. 44, 207 (1989).

[9] T. Ando, Phys. Rev. B 47, 9621 (1993).

[10] Lin-Wang Wang, A. Zunger, Phys. Rev. B 56, 12395 (1997).

[11] I.L. Aleiner, E.L. Ivchenko, Semiconductors 27, 330 (1993) [Fiz. Tekh. Poluprovodn. 27, 594 (1993)].

[12] F. Minami, T. Nakayama, K. Inoue, Jpn. J. Appl. Phys. Suppl. 32-1, 70 (1993).

[13] M. Nakayama, K. Imazawa, K. Suyama, I. Tanaka, H. Nishimura, Phys. Rev. B 49, 13564 (1994).

[14] C. Gourdon, D. Martins, P. Lavallard, E.L. Ivchenko, Yun-Lin Zheng, R. Planel, Phys. Rev. B 62, 16856 (2000).

[15] R.M. Wentzcovitch, M. Cardona, M.L. Cohen, N.E. Christensen, Solid State Commun. 67, 927 (1988)

[16] M. Maaref, F.F. Charfi, D. Scalbert, C. Benoit à la Guillaume, R. Planel, Phys. Status Solidi B 170, 637 (1992). 